\title{
Unitary Amicable Numbers
}

\author{
By Peter Hagis, Jr.
}

\begin{abstract}
Two positive integers are said to be unitary amicable if the sum of the unitary divisors of each is equal to their sum. In this paper a table of such numbers is given, and some theorems concerning them are proved.
\end{abstract}

1. Introduction. In this paper, lower-case letters will be used to denote positive integers, with $p$ and $q$ always representing primes. If $c d=n$ and $(c, d)=1$, then $d$ is said to be a unitary divisor of $n$. We shall use the symbol $\sigma^{*}(n)$ to denote the sum of the unitary divisors of $n$. It is immediate that $\sigma^{*}(1)=1$, while

$$
\sigma^{*}(n)=\left(1+p_{1}^{a_{1}}\right)\left(1+p_{2}^{a_{2}}\right) \cdots\left(1+p_{s}^{a_{1}}\right),
$$

if $n=p_{1}^{a_{1}} p_{2}^{a_{2}} \cdots p_{s}^{a_{2}}$. From (1) we see that $\sigma^{*}(n)$ is a multiplicative function; and $\sigma^{*}(n)=\sigma(n)$ (where $\sigma(n)$ represents the sum of all the positive divisors of $n$ ) if and only if $n$ is square-free. We also obtain the following result from (1).

LEMMA. $\sigma^{*}(n)$ is odd if and only if $n$ is a power of 2 .

Subbarao and Warren [10] have defined $n$ to be a unitary perfect number if $\sigma^{*}(n)=2 n$. Similarly, we shall say that a pair of positive integers $m$ and $n$ are unitary amicable numbers if

$$
\sigma^{*}(m)=\sigma^{*}(n)=m+n .
$$

To the best of my knowledge, such numbers have not been studied, or even defined, before. In this paper, some elementary theorems concerning pairs of unitary amicable numbers are proved and a short table of such pairs is given. Some questions are also posed.

2. Some Theorems. In what follows, $m$ and $n$ are assumed to be members of a pair of unitary amicable numbers. It is obvious that neither $m$ nor $n$ is one.

THEOREM 1. Neither $m$ nor $n$ is of the form $p^{a}$.

Proof. Assume that $n=p^{a}$. Then from (1) and (2), we have $m=1$. This is impossible.

THEOREM 2. $m$ and $n$ are of the same parity.

Proof. Assume the contrary. Then $\sigma^{*}(n)$ is odd and therefore, by our lemma, $n=2^{a}$. This contradicts Theorem 1 .

THEOREM 3. If $m=2^{a} M$ and $n=2^{b} N$ where $b>a>0$ and $M$ and $N$ are odd integers having, respectively, $s$ and $t$ distinct prime divisors, then $s \leqq a$ and $t \leqq a$.

Received November 18, 1970, revised March 1, 1971.

AMS 1969 subject classifications. Primary 1002, 1005.

Key words and phrases. Unitary divisors, amicable numbers, unitary amicable numbers.

Copyright @ 1971 , American Mathematical Society 
Proof. We see immediately that $m+n=2^{a} r$ where $r$ is odd. If $M=p_{1}^{a_{1}} \cdots p_{a}^{a_{a}}$, then from (1) and (2), $2^{a} r=\left(1+2^{a}\right)\left(1+p_{1}^{a_{1}}\right) \cdots\left(1+p_{s}^{a^{a}}\right)$. Since each factor, except the first, on the right-hand side is even, we have $s \leqq a$. By the same argument $t \leqq a$.

COROLlaRY 3.1. If $m=2 M$ and $n=2^{b} N$ where $b>1$, then $M=p^{o}$ and $N=q^{h}$.

COROLlARY 3.2. If $M$ and $N$ are squares and $b>a$, then $s=t=a$.

COROLlARY 3.3. If $M$ and $N$ are squares and $b=a$, then $s=t=a+1$.

(For Corollary 3.2, recall that $1+p^{2 c}=2+4 k$ if $p$ is odd; for Corollary 3.3 recall that $u^{2}+v^{2}=2+8 k$ if $u$ and $v$ are odd.)

THEOREM 4. If $m=2 M$ and $n=2 N$, where $(10, M N)=1$, then $10 m$ and $10 n$ also constitute a pair of unitary amicable numbers.

Proof. From the multiplicative property of $\sigma^{*}$, we have

$$
\begin{aligned}
\sigma^{*}(10 m) & =\sigma^{*}(4) \sigma^{*}(5) \sigma^{*}(M)=30 \sigma^{*}(M) \\
& =10 \cdot \sigma^{*}(2) \sigma^{*}(M)=10 \sigma^{*}(m)=10 m+10 n .
\end{aligned}
$$

In the same way, $\sigma^{*}(10 n)=10 m+10 n$.

A similar argument establishes Theorem 5, while our final theorem follows from the remark made just after (1).

THEOREM 5. If $m=12 M$ and $n=12 N$ where $(6, M N)=1$, then the pair $18 M$ and $18 N$ are also unitary amicable numbers.

THEOREM 6. If $m$ and $n$ are both square-free, then the pair $m, n$ is unitary amicable if and only if the pair $m, n$ is amicable.

It is, perhaps, of some interest to compare the above results with our state of knowledge concerning (ordinary) amicable numbers.

Remark 1. Whether it is possible for a number of the form $p^{a}$ to be one of a pair of amicable numbers is not known. It is known that an affirmative answer would imply $p^{a}>10^{1500}$ and $a>1400$ (see [6]).

Remark 2. Whether or not a pair of amicable numbers of opposite parity exists is also an open question. Some necessary conditions for the existence of such a pair may be found in [5] and [7].

Remark 3. Theorem 3 is false for amicable numbers. A counterexample is provided by the pair $2 \cdot 5 \cdot 11^{2}$ and $2^{5} \cdot 37$.

Remark 4. To my knowledge, no results analogous to Theorems 4 and 5 are known concerning even amicable numbers. However, the referee has pointed out that many "isomeric" pairs of odd amicable numbers exist (for example, see the pairs 60 and 61, 65 and 66, 138 and 139 in [3]), and that theorems similar to Theorems 4 and 5 could be (although apparently they have not been) stated concerning them.

3. A List of Unitary Amicable Pairs. Using the CDC 6400 at the Temple University Computing Center, a search was made for all unitary amicable pairs $m, n$ such that $m<n$ and $m \leqq 10^{6}$. The search required about two hours and ten minutes of computer time, and 19 pairs were found. These comprise the first 19 entries in the list at the end of this section. Of these, three (pairs 16, 18, 19) satisfied the hypotheses of Theorem 5 and had $m>666666$. An application of this theorem yielded the next three pairs in our list. The eighth pair, 142310 and 168730 , is squarefree and therefore, by Theorem 6, is amicable. A search of the published pairs of amicable numbers (see [1], [2], [3], [4], [8], [9] and [11]) yielded 76 square-free pairs 
including the one just mentioned. Nine of these pairs are odd, the smallest being 8619765 and 9627915 . Of the remaining 67 pairs, eleven satisfy the conditions of Theorem 4 and therefore, upon multiplication by 10, yield "new" unitary pairs. These comprise the last eleven pairs in our list. Thus, a total of 108 unitary amicable number pairs are known to date, 76 being square-free and therefore simultaneously amicable, the remaining 32 being "new".

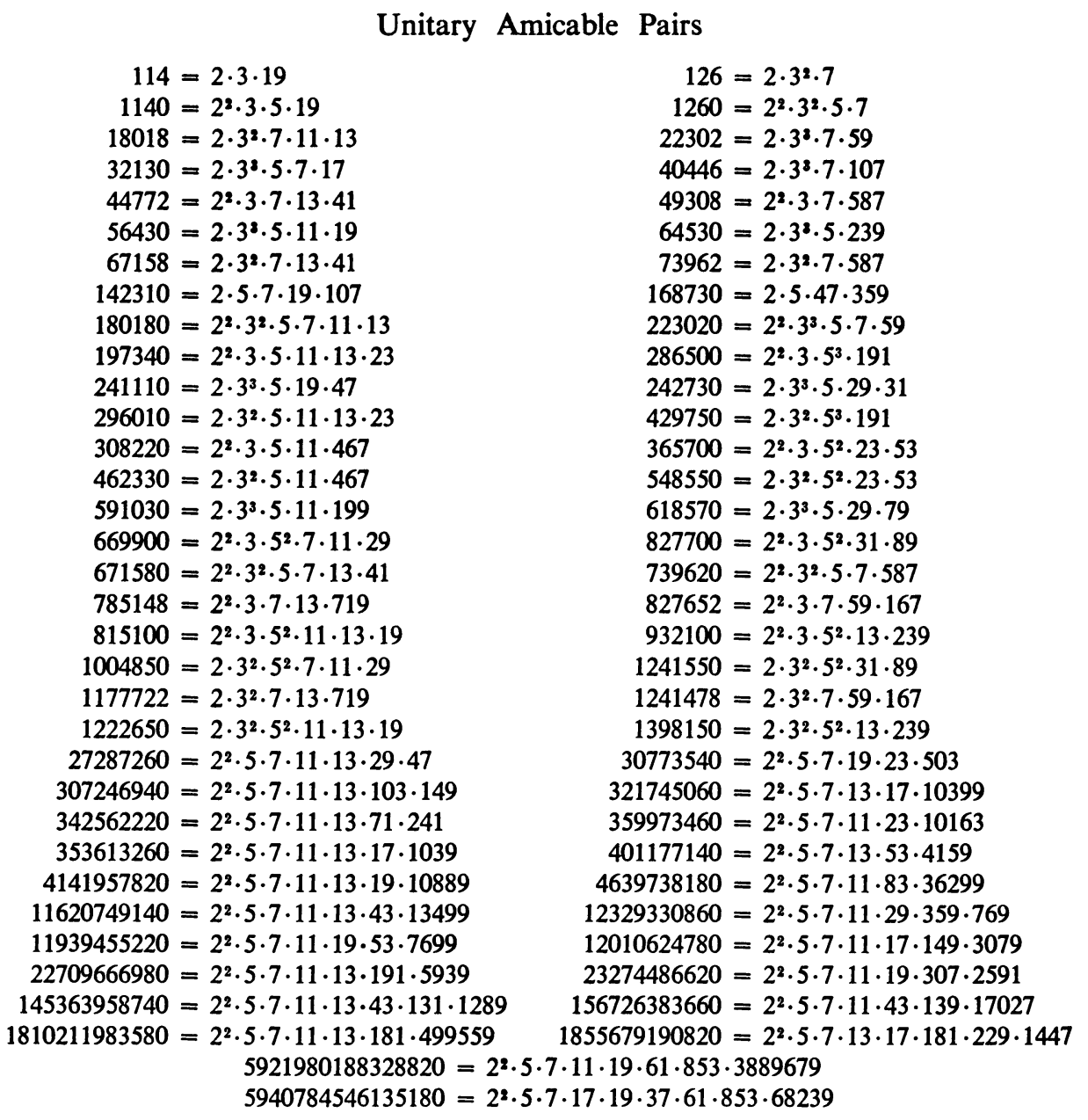

4. Some Questions. An examination of the list in Section 3 suggests the following questions.

Question 1. Are there an infinite number of unitary amicable numbers?

Question 2. Does there exist a relatively prime pair of unitary amicable numbers?

Question 3. Is every odd pair of unitary amicable numbers simultaneously amicable?

Question 4. If $m=2^{a} M$ and $n=2^{b} N$, where $M N$ is odd, is it always the case that $a=b$ ?

Question 5. Are there an infinite number of "twin" pairs of unitary amicable 
numbers (such as the tenth and eleventh pairs or the twenty-eighth and twenty-ninth pairs in our list) with the property that $m_{1}+n_{1}=m_{2}+n_{2}$ ?

I would conjecture that the answer to the first question is "Yes", while the answer to the second, third and fourth questions is "No". I have no opinion to express on the final question.

Department of Mathematics

Temple University

Philadelphia, Pennsylvania 19122

1. J. Alanen, O. ORe \& J. Stemple, "Systematic computations on amicable numbers," Math. Comp., v. 21, 1967, pp. 242-245. MR 36 \#5058.

2. P. Bratley \& J. McKay, "More amicable numbers," Math. Comp., v. 22, 1968, pp. 677-678. MR 37 \#1299.

3. E. B. Escotr, “Amicable numbers," Scripta Math., v. 12, 1946, pp. 61-72. MR 8, \#5158.

4. M. García, "New amicable pairs," Scripta Math., v. 23, 1957, pp. 167-171. MR 20

5. A. A. Giola \& A. M. VaIdya, "Amicable numbers with opposite parity," Amer. Math. Monthly, v. 74, 1967, pp. 969-973. MR 36 \#3711.

6. H. -J. KANOLD, "Uber befreundete Zahlen. I," Math. Nachr., v. 9, 1953, pp. 243-248. MR 15, 506.

7. H. -J. KANOLD, "Uber befreundete Zahlen. II," Math. Nachr., v. 10, 1953, pp. 99-111. MR 15, 506.

8. E. J. LEE, "Amicable numbers and the bilinear diophantine equation," Math Comp., v. 22, 1968, pp. 181-187. MR 37 \#142.

9. P. Poulet, “43 new couples of amicable numbers," Scripta Math., v. 14, 1948, p. 77. 10. M. V. Subbarao \& L. J. Warren, "Unitary perfect numbers," Canad. Math. Bull., v. 9, 1966, pp. 147-153 MR 33 \#3994. 429.

11. H. CoHEN, "On amicable and sociable numbers," Math. Comp., v. 24, 1970, pp. 423- 